\title{
Homing et mobilisation des cellules hématopoïétiques: un dédale très bien balisé...
}

Un apect fascinant du fonctionnement de l'organisme est l'efficacité avec laquelle les cellules circulantes trouvent leur chemin vers l'organe cible dans le dédale des vaisseaux. La séquence d'événements guidant lymphocytes et polynucléaires vers le tissu lésé lors de la réaction immune ou inflammatoire est maintenant bien disséquée, et implique chimiokines, ligands endothéliaux et récepteurs correspondants sur les effecteurs circulants [1]. Curieusement, la migration des cellules hématopoiétiques vers (homing) et hors de (mobilisation) la moelle osseuse a été jusqu'à maintenant beaucoup moins explorée. Pourtant c'est une étape fondamentale de la transplantation hématologique, et ce pour deux raisons: (1) les cellules du donneur sont injectées par voie intraveineuse, et (2) les cytokines utilisées en thérapeutique (G-CSF par exemple) induisent la «mobilisation» des cellules souches hématopoiétiques hors de la moelle [2]. Deux articles très complémentaires publiés par le même groupe ont évalué le rôle des sélectines et de VCAM (vascular cell adhesion molecule) dans le homing des cellules souches hématopoiétiques. La première approche a été faite in vivo [3]: la cinétique de l'immobilisation des cellules hématopoiétiques (marquées par un fluorochrome) au niveau des vaisseaux irriguant la moelle osseuse a été filmée au niveau de la boîte cranienne car la table osseuse y est très fine. La seconde approche [4] a analysé le homing a posteriori, en jugeant de l'efficacité de la greffe chez des receveurs irradiés sur le contenu de leur moelle osseuse en progéniteurs hématopoïétiques issus du donneur 14 heures après la greffe, ou sur leur survie 20-30 jours après la greffe. Dans les deux approches, l'analyse a été facilitée par l'utilisation de souris dont les gènes des sélectines $\mathrm{E}$ et $\mathrm{P}$ ont été invalidés (l'invalidation de VCAM est létale). Les deux études s'accordent à conclure que le homing des cellules hématopoḯtiques fait intervenir les sélectines $\mathrm{E}$ et $\mathrm{P}$ et VCAM. L'analyse dynamique in situ montre que, comme pour les leucocytes, la première étape du homing des progéniteurs dans la moelle fait intervenir un processus de rolling sur VCAM et les sélectines, précédant l'adhérence ferme puis l'extravasation. Rappelons que, dans la moelle, VCAM et les sélectines sont spontanément exprimés à la surface des cellules endothéliales, contrairement aux autres territoires vasculaires où ces molécules sont induites en réponse à un stimulus endothélial. Cependant, il y a redondance, et si chacune de ces molécules contribue au homing, aucune n'est indispensable. Le rôle de VCAM par exemple, n'est décelable qu'en l'absence des sélectines. On pouvait s'en douter puisque les souris aux gènes des sélectines $\mathrm{E}$ et $\mathrm{P}$ invalidés ont une hématopoièse active. L'histoire n'en est qu'à son début car il est difficile d'expliquer la spécificité du homing dans la moelle par la seule intervention de molécules (sélectines, VCAM) dont la distribution est ubiquitaire. D'autres partenaires, en particulier des chimiokines, peut-être spécifiques des différents organes cibles comme la moelle, seront sûrement identifiés. On peut aussi parier que la spécificité du homing est conférrée par la combinaison des couples ligandsrécepteurs, et par leur état d'activation, plutôt que par la mise en jeu de molécules spécifiques d'un tissu ou d'un organe donné.

L.C.

1. Néel D, Aubery M, Derappe C. Glycobiologie et cellules sanguines. Med Sci 1992; 8: 233-8.

2. Thuran A. Mobilisation sélective de cellules souches hématopoiétiques dans les hémopathies malignes : modèle de la leucémie myéloïde chronique. Med Sci 1998; 14: 198-202.

3. Mazo IB, Guttierez-Ramos JC, Frenette P, Hynes R, Wagner D, Von Andrian, U. Hematopoietic progenitor cell rolling in bone marrow microvessels: parallel contributions by endothelial selectins and vascular cell adhesion molecule 1. J Exp Med 1998; 188: 465-74.

4. Frennette P, Subbarao S, Mazo IB, von Andrian U, Wagner D. Endothelial selectins and vascular cell adhesion molecule-1 promote hematopoietic progenitor homing to bone marrow. Proc Natl Acad Sci USA 1998; 95 : 14423-8. 